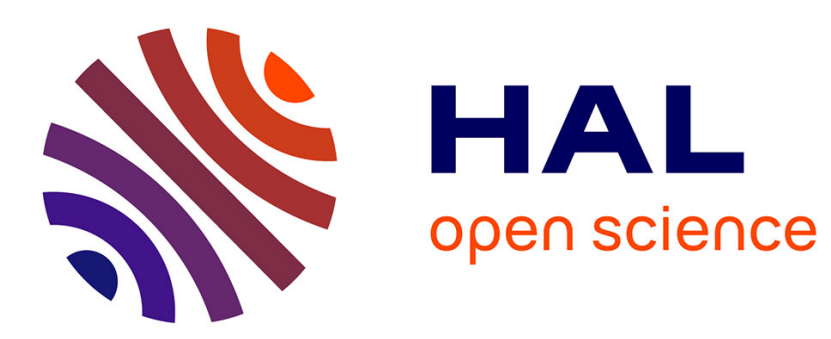

\title{
Stick-slip waves between elastic and rigid half-spaces
}

Huy Duong Bui, Abdelbacet Oueslati

\section{To cite this version:}

Huy Duong Bui, Abdelbacet Oueslati. Stick-slip waves between elastic and rigid half-spaces. Journal of Applied Mathematics and Mechanics, 2012, 76 (5), p. 611-620. 10.1016/j.jappmathmech.2012.11.003 . hal-00787848

\section{HAL Id: hal-00787848 \\ https://hal-polytechnique.archives-ouvertes.fr/hal-00787848}

Submitted on 13 Feb 2013

HAL is a multi-disciplinary open access archive for the deposit and dissemination of scientific research documents, whether they are published or not. The documents may come from teaching and research institutions in France or abroad, or from public or private research centers.
L'archive ouverte pluridisciplinaire HAL, est destinée au dépôt et à la diffusion de documents scientifiques de niveau recherche, publiés ou non, émanant des établissements d'enseignement et de recherche français ou étrangers, des laboratoires publics ou privés. 


\title{
Stick-slip waves between elastic and rigid half-spaces
}

\author{
H.D. Bui ${ }^{a, b}$, A. Oueslati $^{c}$ \\ ${ }^{a}$ Laboratory of Solid Mechanics, Ecole Polytechnique, 91128 Palaiseau, France \\ ${ }^{b}$ UME Mecanique, ENSTA, 91128 Palaiseau France \\ ${ }^{c}$ Université de Lille 1, Laboratoire de Mécanique de Lille/CNRS UMR 8107, 59655 \\ Villeneuve d'Ascq, France
}

\begin{abstract}
This paper is devoted to the construction of analytic solution of stick-slip waves crossing the interface between an elastic half-space and a rigid one under unilateral contact and Coulomb friction. The method of solution is based on the analytic continuation method of Radok's complex potentials within the framework of steady elastodynamical problems. The governing equations combined with the boundary conditions are reduced to a RiemannHilbert problem with discontinuous coefficient and closed-form expressions of the solution are derived. It is found that the existence of solutions depends on the additional velocity $V^{*}$ which is related to the longitudinal elongation. If $V^{*}$ is ignored $\left(V^{*}=0\right)$ then there is no solution, if not $\left(V^{*} \neq 0\right)$ then it is possible to construct weakly singular solutions satisfying all stick-slip conditions except over a narrow zone where the waves exhibit a crack-like behaviour.
\end{abstract}

Key words: Stick-slip waves, Unilateral contact, Coulomb friction, Riemann-Hilbert problem, Crack-like behaviour

\section{Introduction}

This paper is dedicated to Professor L.A. Galin whose scientific contributions are exceptionally rich and widely recognized in contact and friction mechanics [1-5]. Within this field, we present here some of our results and

Email addresses: hdb@lms.polytechnique.fr (H.D. Bui ${ }^{a, b}$, A. Oueslati ${ }^{c}$ ), abdelbacet.oueslati@univ-lille1.fr (H.D. Bui ${ }^{a, b}$, A. Oueslati $^{c}$ ) 
open questions related to the problem of stick-slip waves between an elastic half plane and a rigid one [6].

During the two last decades, an important issue emerges in the area of elastodynamic problems involving frictional contact following Coulomb's law, namely the friction-induced vibrations under the form of stick-slip waves. Such cyclic responses, generally accompanied with noise emittence, are closely related to the dynamic bifurcation from the steady response to a cyclic vibration in the sense of Poincar-Hopf bifurcation [7]. In literature, first studies of stick-slip focused on discrete systems, typically the Van-der Pol oscillator. For such mechanical systems, the formation of stick-slip motion is attributed to a static coefficient of friction higher than a kinematic one or to the decay of the kinematic coefficient with the sliding velocity.

Recent investigations of the steady sliding contact between dissimilar elastic half-spaces or between a semi-infinite elastic solid and a rigid one showed that the steady state is dynamically unstable for a constant friction coefficient [8-11]. Adams [11] suggested that this dynamic instability is related to the destabilization of the so-called interfacial slip waves. Such waves exist in frictionless contact and are called also the "generalized Rayleigh waves" because they propagate with the Rayleigh wave celerity. Later Ranjith et al [12] demonstrated that, for material combinations where the generalized Rayleigh wave exists, the steady sliding with Coulomb friction is dynamically unstable for an arbitrarily small values of the friction coefficient.

Although the mechanism of flutter instability of friction is now well understood [11, 12], only few works are devoted to the construction of analytic solution of self-excited vibrations for continuum media. Without being exhaustive, we note that first solutions of stick-slip or stick-slip-separation waves travelling along the interface between two identical elastic half-spaces, were proposed by Comninou [13, 14]. Later, Freund [15] pointed out a similarity feature between the Comninou's waves and the propagation of interface cracks and showed that the encountered singularities imply energy sources and sinks. Hence, Comninou's solutions were controversial and not physical. For these same reasons, Adams [16] didn't succeed to construct an interfacial stick-slip waves between two dissimilar half-spaces, however, in [17] a family of slip pulse propagating with the celerity of generalized Rayleigh waves is successfully built. Adda-Bedia et al [18] studied the existence of a steadystate slip pulse on finite size between two dissimilar materials. It has been found found that solutions showing a crack-like behavior exist with an arbitrary slip velocity and an arbitrary slip length. For bounded solids, to our 
knowledge, the only semi-analytic stick-slip and stick-slip-separation waves are derived in [19-21] in view of the study of two-dimensional brake-like system.

\section{Formulation of the problem}

Consider an elastic solid, with shear modulus $G$ and waves velocities $c_{1}$, $c_{2}$ occupying the lower half space $\Omega^{-}$and sliding against the upper rigid halfspace $\Omega^{+}$which moves to the right with velocity $V$, as shown in Fig.1, in plane strain conditions. Unilateral contact and Coulomb friction with constant friction coefficient $\mu$ are assumed. It is emphasized that the interfacial or local coefficient of friction $\mu$ is the ratio of shear to normal contact pressure at the interface which would cause local slipping to occur [17]. The elastic body is subjected to remote constant stresses $\tau_{y y}^{*}<0, \tau_{x y}^{*}>0$ such that $\tau_{x y}^{*}=-\mu^{*} \tau_{y y}^{*}$, with $\mu^{*}<\mu$.

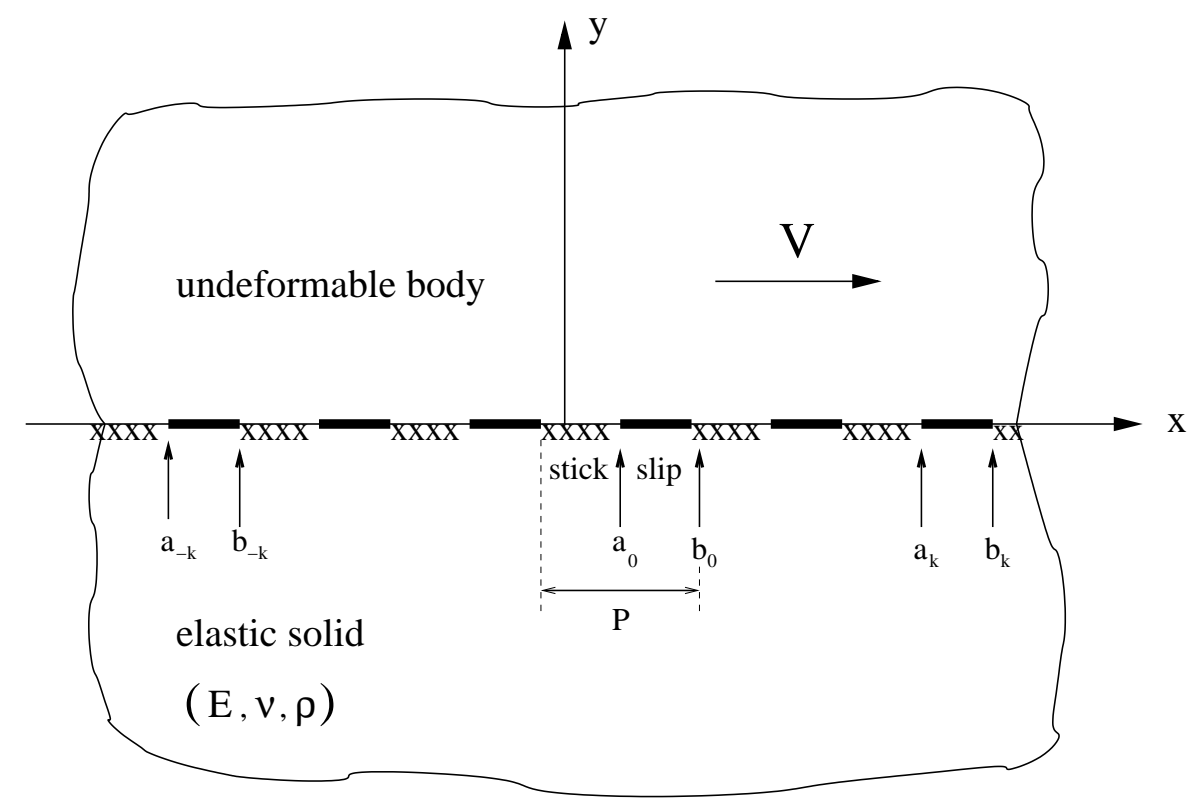

Figure 1: A rigid body sliding on an elastic half-space

Suppose that the interface is entirely a stick zone, then the velocity of points of the interface $y=0^{-}$, is $\dot{U}_{x}=V$. Since the shear stress grows indefinitely, the interface will no longer adhere to the upper non-deformable solid. 
On the other hand, suppose that the interface $y=0^{-}$is entirely a sliding one, with the constant static stress $\left(\tau_{x y}^{*}>0, \tau_{y y}^{*}<0\right)$. Thus the elastic body is at rest under static stress, $\dot{U}_{x}=0$. The slip condition of the Coulomb's friction law is not satisfied. Therefore both conditions on the velocity and Coulomb's friction law are incompatible. Thus, we investigate here the possibility of relative motion of the two bodies, due to the existence of periodic stick and slip regions which propagate along the interface with some wave speed $c$. In this case the quantity $\mu^{*}$ may be interpreted as the apparent coefficient of friction, since sliding occurs with that ratio of applied shear to normal traction $[17,18,22]$.

We assume that the periodic stick-slip wave consists in a stick region plus a slip one indefinitely repeated as shown in Fig.1.

Let us denote by $S L$ the set of the slip segments

$$
S L=\cdots\left[a_{-k}, b_{-k}\right] \bigcup \cdots\left[a_{0}, b_{0}\right] \bigcup \cdots\left[a_{k}, b_{k}\right] \bigcup \cdots, \quad k \in \mathbb{N}
$$

The remainder part of contact interface is the stick zone and will be refereed by $S T$. The infinite axis $y=0$ is oriented in the direction of increasing $x$ and the following notation will be frequently used: $S L^{+}=\lim _{y \rightarrow 0^{+}} S L$ and $S L^{-}=\lim _{y \rightarrow 0^{-}} S L$. In the same way, one defines $S T^{+}$and $S T^{-}$.

If we fix the origin of coordinate frame at $b_{-1}=0$, we can simply denote any particular slip zone $\left[a_{i}, b_{i}\right]$ by its generic segment $\left[a \equiv a_{0}, b \equiv b_{0}\right]$ and any stick zone $\left[b_{k-1}, a_{k}\right]$ by its generic segment $\left[b_{-1}=0, a \equiv a_{0}\right]$. We note $P=\left[0, a_{0}\right] \cup\left[a_{0}, b_{0}\right]$.

Together with a fixed frame coordinates $(X, Y)$, we shall use also moving coordinates with the algebraic velocity $c$. Later we shall se that $c<0$. Capital letters $U_{i}, \Sigma_{i j}$ are for coordinates in fixed frame, small letters for coordinates in moving frame. Hence $X=x+c t$ and $Y=y$. Material derivative in steady state case $\left(\frac{\partial g}{\partial t}=0\right)$ is denoted by a dot, $\frac{d g}{d t} \equiv \dot{g}=-c g_{, x}$.

In the present work, it is reasonable to assume that the periodic stick-slip regime occurs under the condition discussed above $\dot{U}_{x}=0$ which is satisfied, not locally in the whole interface, but only in the homogenized sense. That is the "mean" value of the velocity over a stick-slip period $[0, b]$ vanishes

$$
\left\langle\dot{U}_{x}\right\rangle=0
$$

where $\langle f\rangle=\frac{1}{|P|} \int_{P} f d x$. 
This vanishing mean value ensures that the stress field of the lower elastic body does not blow up in time.

In the steady state case, $\mathrm{Eq}(1)$ is equivalent to $\left\langle-c U_{x, x}\right\rangle=0$ or to the periodicity condition

$$
U(b)=U(0)
$$

Condition $\dot{U}_{x}=V$ is only satisfied in the stick zones $S T$, while the sliding condition $\Sigma_{x y}[U]=-\mu \Sigma_{y y}[U]$ is only satisfied in the slip zones $S L$. The incompatibility between boundary conditions is relaxed through homogenization so that the effective or homogenized "friction" coefficient $\mu^{*}$ can be smaller that the true one.

Let the material velocity in the elastic body at interface be decomposed into the sum

$$
\dot{U}=V^{*}+\dot{u}=V^{*}-c u_{x, x}
$$

where $V^{*}$ is some velocity defined hereafter. We shall show that the presence of longitudinal elongation $\varepsilon_{x x}^{*}$ enables to solve the periodic stick-slip problem. If this elongation is ignored by setting $\varepsilon_{x x}^{*}=0$ in the equation, we will show that there is no solution. From elastic law, the elongation in the $x$-direction is

$$
\varepsilon_{x x}^{*}=u_{x, x}^{*}=-\frac{\nu(1+\nu)}{E} \tau_{y y}^{*}>0
$$

Notice that the mode deformation expressed by (4) is derived from Hooke's law with constant stress $\tau_{x y}^{*}=-\mu \tau_{y y}^{*}$ and $\tau_{x x}^{*}=0$. In the present work the most important stresses are $\left(\tau_{y y}^{*}, \tau_{x y}^{*}\right)$ since they are involved in the friction law. The analysis and the generality of the results are not affected and remain valid if one takes $\tau_{x x}^{*} \neq 0$.

The velocity $V^{*}$ is defined hereafter by

$$
V^{*}=-c u_{x, x}^{*}=c \frac{\nu(1+\nu)}{E} \tau_{y y}^{*}
$$

Note that $V^{*}$ is positive because $c<0$ as it will be shown hereafter.

$\mathrm{Eq}(5)$ establishes a relationship between $\tau_{y y}^{*}, c$ and $V^{*}$. Hence, the principal unknowns are the wave celerity $c$ and $b$ for prescribed stresses and $V^{*}$.

Let $\sigma_{x y}, \sigma_{y y}$ and $\sigma_{x x}$ be the additional stresses correspondent to the perturbed stick-slip motion. The solution satisfies the following conditions

- Active contact along the interface $y=0$ :

$$
u_{y}=0
$$


- Material velocity:

$$
\dot{U} \equiv-c u_{x, x}+V^{*}=V \quad \text { in the stick region } S T
$$

- Sliding condition:

$\Sigma_{x y}[U] \equiv \sigma_{x y}+\tau_{x y}^{*}=-\mu\left(\sigma_{y y}+\tau_{y y}^{*}\right) \equiv-\mu \Sigma_{y y}[U]$ in the slip region $S L$

- The following inequality:

$$
\left|\Sigma_{x y}[U]\right|<\mu\left|\Sigma_{y y}[U]\right| \quad \text { in the stick zone } S T
$$

- $\sigma_{x y}$ and $\sigma_{y y}$ behaves like

$$
\sigma_{i j}=O\left(1 /\left(x^{2}+y^{2}\right)\right) \quad \text { at infinity }
$$

We search $c, \nabla u, \sigma_{x y}, \sigma_{y y}$ periodic satisfying relations (6-10). An addition condition comes from the periodicity of fields. Note that even if the following fields $\dot{U}, U$ (displacement), $\nabla u, \sigma$ are periodic, the field $u_{x}$, is not. A counter-example can be seen in Fig.2 for the periodic field (continuous line) $u_{x, x}=1$ in $[0,1], u_{x, x}=-1$ in $[1, b \neq 0]$ and the non-periodic continuous field $u_{x}$ (doted line).

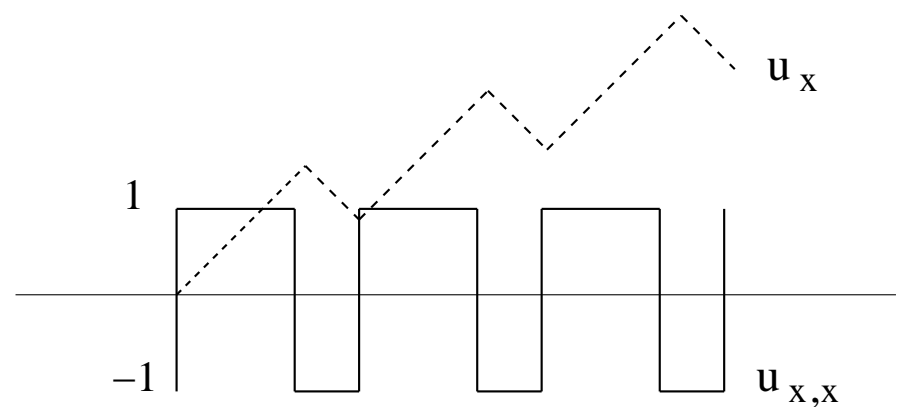

Figure 2: The gradient field $u_{x, x}$ is periodic (continuous line) however the displacement $u_{x}$ is non-periodic (doted line)

From Eq(3) we get

$$
-c U_{x, x}=-c u_{x, x}+V^{*}
$$


Assuming the periodicity condition for the total displacement $U(x)$

$$
U_{x}(b)-U_{x}(0)=0
$$

we get $-c\left(U_{x}(b)-U_{x}(0)\right)=-c\left(u_{x}(b)-u_{x}(0)\right)+V^{*} b=0$ and thus

$$
u_{x}(b)-u_{x}(0)=\frac{b V^{*}}{c}
$$

Thus, $u_{x}(x, 0)$ is not periodic. The constant $-V^{*} / c$ has been introduced as the applied elongation $\varepsilon_{x x}^{*}$. Another interpretation of (13) is the sliding displacement over a period. Remark that Eq.(1) shows that the "mean perturbed velocity" $\langle\dot{u}\rangle$ over a period of length $b$ is opposite to $V^{*}$. Thus we recover $(13)$

$$
\langle\dot{u}\rangle=-\frac{c}{b}\left(u_{x}(b)-u_{x}(0)\right)=-V^{*}
$$

Another interpretation of $\mathrm{Eq}(1)$ is to impose the overall "mean" strain $U_{x, x}$ equal to zero, or to require the "mean" strain $\left\langle u_{x, x}\right\rangle$ be opposite to the applied strain $u_{x, x}^{*}=-\frac{V^{*}}{c}$.

Let us now recall the steady elastodynamic equations within the framework of homogenous and isotropic elasticity under the plane strain hypothesis. In the moving frame $(O x y)$ attached to the propagating stick-slip wave $(x=X-c t, \quad y=Y)$, one introduces the Radok complex variables

$$
z_{1}=x+i \beta_{1} y \quad ; \quad z_{2}=x+i \beta_{2} y
$$

where $\beta_{1}=\sqrt{1-\frac{c^{2}}{c_{1}^{2}}}, \beta_{2}=\sqrt{1-\frac{c^{2}}{c_{2}^{2}}}, i=\sqrt{-1}$ is the imaginary unit number and $c_{1}, c_{2}$ stand for the celerity of longitudinal and shear waves defined respectively by

$$
c_{1}=\sqrt{\frac{\lambda+2 G}{\rho}}, \quad c_{2}=\sqrt{\frac{G}{\rho}}
$$

$G$ is the shear modulus, $\lambda$ denotes the Lamé's coefficient and $\rho$ is the mass density.

The displacement and stress fields are given in terms of tow complex poten- 
tials $\phi_{1}$ and $\phi_{2}$ as follows [23]

$$
\begin{aligned}
& u_{x}=-\frac{1}{G} \Re e\left(\phi_{1}\left(z_{1}\right)+\frac{1+\beta_{2}^{2}}{2} \phi_{2}\left(z_{2}\right)\right) \\
& u_{y}=\frac{1}{G} \Im m\left(\beta_{1} \phi_{1}\left(z_{1}\right)+\frac{1+\beta_{2}^{2}}{2 \beta_{2}} \phi_{2}\left(z_{2}\right)\right) \\
& \sigma_{x x}=-2 \Re e\left(\frac{2 \beta_{1}^{2}-\beta_{2}^{2}+1}{2} \phi_{1}^{\prime}\left(z_{1}\right)+\frac{1+\beta_{2}^{2}}{2} \phi_{2}^{\prime}\left(z_{2}\right)\right) \\
& \sigma_{y y}=\left(1+\beta_{2}^{2}\right) \Re e\left(\phi_{1}^{\prime}\left(z_{1}\right)+\phi_{2}^{\prime}\left(z_{2}\right)\right) \\
& \sigma_{x y}=2 \Im m\left(\beta_{1} \phi_{1}^{\prime}\left(z_{1}\right)+\frac{\left(1+\beta_{2}^{2}\right)^{2}}{4 \beta_{2}} \phi_{2}^{\prime}\left(z_{2}\right)\right)
\end{aligned}
$$

where $\Re e(Z)$ and $\Im m(Z)$ represent respectively the real and the imaginary part of the complex number $Z$.

The present study focus on the subsonic waves i.e. $c<c_{2}$. Hence, $\beta_{1}>0$ and $\beta_{2}>0$.

\section{Construction of the solution}

\subsection{Basic equations}

Following Bui et al [24, 25], the construction method of the solution is based on the displacement continuation. From the condition $u_{y}=0$ along the contact interface (real axis where $z_{1}=z_{2}=z=x \pm i 0$ ) and equation (16) one obtains

$$
\beta_{1} \phi_{1}(z)+\frac{\left(1+\beta_{2}^{2}\right)}{2 \beta_{2}} \phi_{2}(z)=0
$$

This equation suggests the following definition for the function $\phi_{2}$ in the physical domain by

$$
\phi_{2}\left(z_{2}\right):=-\frac{2 \beta_{1} \beta_{2}}{\left(1+\beta_{2}^{2}\right)} \phi_{1}\left(z_{2}\right)
$$


Substitution of (21) in (15-19) results in the following equations on the interface $(y=0)$ where $z_{1}=z_{2}=z$

$$
\begin{aligned}
& u_{x}=-\frac{\left(1-\beta_{1} \beta_{2}\right)}{G} \Re e\left(\phi_{1}(z)\right) \\
& \sigma_{y y}=\left(1+\beta_{2}^{2}-2 \beta_{1} \beta_{2}\right) \Re e\left(\phi_{1}^{\prime}(z)\right) \\
& \sigma_{x x}=\left(\left(-1+\beta_{2}^{2}\right)+2 \beta_{1}\left(\beta_{2}-\beta_{1}\right)\right) \Re e\left(\phi_{1}^{\prime}(z)\right) \\
& \sigma_{x y}=\beta_{1}\left(1-\beta_{2}^{2}\right) \Im m\left(\phi_{1}^{\prime}(z)\right)
\end{aligned}
$$

Hence, all mechanical fields are determined through the knowledge of the function $\phi_{1}$ and its derivative $\phi_{1}^{\prime}$.

In the sequel, unless stated otherwise, the notation $\Phi(z):=\phi_{1}^{\prime}(z)$ will be used.

We shall search for a complex solution under the form of a Cauchy integral with a distribution $f(t)$

$$
\Phi(z)=\frac{1}{2 i \pi} \int_{a}^{b} \frac{f(t)}{t-z} d t
$$

where $z=x+i y$.

It is useful to underline that if $f(t)$ is real then the conjugate function of $\Phi$ is given, for $z$ on the real axis, by

$$
\bar{\Phi}(z)=-\frac{1}{2 i \pi} \int_{a}^{b} \frac{f(t)}{t-z} d t=-\Phi(z)
$$

Note that (27) may remains valid for some complex functions $f(t)$. It will be the case for the solution in the slip zone as it will be shown hereafter.

\subsection{Solution in the slip zone}

The sliding state expressed by equation (8) may be written as

$$
\sigma_{x y}+\tau_{x y}^{*}=-\mu\left(\sigma_{y y}+\tau_{y y}^{*}\right) \Rightarrow \sigma_{x y}+\mu \sigma_{y y}=-\tau_{x y}^{*}-\mu \tau_{y y}^{*}:=T^{*}
$$

The stress $T^{*}$ can be written as $T^{*}=\mu^{*} \tau_{y y}^{*}-\mu \tau_{y y}^{*}=\left(\mu^{*}-\mu\right) \tau_{y y}^{*}>0$. It is positive because $\mu^{*}<\mu$ and $\tau_{y y}^{*}<0$. 
By setting $\gamma_{1}=\beta_{1}\left(1-\beta_{2}^{2}\right)>0$ and $\gamma_{2}=1+\beta_{2}^{2}-2 \beta_{1} \beta_{2}$ and by virtue of $\bar{\Phi}^{+}(z)=-\Phi^{+}(z)$, Eq.(28) becomes

$$
-g \Phi^{-}(z)+\Phi^{+}(z)=\frac{2 i T^{*}}{\gamma_{1}-i \mu \gamma_{2}}:=f_{2}(t)
$$

where

$$
f_{2}(t)=\frac{2 i T^{*}}{\gamma_{1}-i \mu \gamma_{2}}
$$

is a constant and

$$
g=-\frac{\gamma_{1}+i \mu \gamma_{2}}{\gamma_{1}-i \mu \gamma_{2}}
$$

It is usefull to notice that the coefficient $g$ can be expressed in a different manner

$$
g=-e^{2 i \pi \alpha}=e^{2 i \pi\left(\alpha+\frac{1}{2}\right)}, \quad|g|=1
$$

with

$$
\tan (\alpha \pi)=\mu \frac{\gamma_{2}}{\gamma_{1}}
$$

Equation (29) is called the Riemann-Hilbert problem and has a closed form solution [26]. The appropriate $\mathbf{P}$-periodic solution of the homogenous RiemannHilbert problem has the following form

$$
X(z)=\prod_{k=-\infty}^{+\infty}(z-(a+k b))^{\frac{1}{2}-\alpha}(z-(k+1) b)^{-\frac{1}{2}+\alpha}
$$

involving product of holomorphic functions with a cut along the slip segments $S L$. Observe that $X(z)$ is regular at $a_{k}=a+k b$ and weakly singular at $b_{k}=(k+1) b .^{1}$

It easily seen that

$$
X(z)=\lim _{N \rightarrow+\infty} \underbrace{\sum_{k=-N}^{+N}(z-(a+k b))^{\frac{1}{2}-\alpha}(z-(k+1) b)^{-\frac{1}{2}+\alpha}}_{X^{N}(z)}=\lim _{N \rightarrow+\infty} X^{N}(z)
$$

\footnotetext{
${ }^{1}$ Regular functions at $a_{k}$ and $b_{k}$ are not considered for the construction of the solution because the infinite product $X(z)$ is divergent at infinity and thus the proposed approach does not work.
} 
It can be proved for a fixed $z$, that the sum $e$ of the remaining terms is of order $e=O(1 / N)$. Thus $X^{N}(z)$ tends absolutely to a limit, denoted by $X(z)$ when $N \rightarrow \infty$.

Following Mushkhelishvili [26], the general solution of (29) has the form

$$
\Phi^{s l i p}(z)=\frac{f_{2}}{2 i \pi} X(z)\left\{\int_{S L^{+}} \frac{1}{X\left(t^{+}\right)} \frac{d t}{t-z}+C_{2}\right\}
$$

where $C_{2}$ is an arbitrary constant. Besides, it is easy to prove that (36) satisfies $\bar{\Phi}(z)=-\Phi(z)$.

\subsection{Solution in the stick zone}

Equating the horizontal velocity in stick zone to $V-V^{*}, u_{x, x}=\frac{\partial u_{x}}{\partial x}=-\frac{\left(V-V^{*}\right)}{c}$, and knowing that $\frac{\partial(\cdot)}{\partial x}=\frac{\partial(\cdot)}{\partial z}+\frac{\partial(\cdot)}{\partial \bar{z}}$ we obtain

$u_{x, x}=-\frac{\left(1-\beta_{1} \beta_{2}\right)}{2 G}\{\Phi(z)+\bar{\Phi}(\bar{z})\}=-\frac{\left(1-\beta_{1} \beta_{2}\right)}{2 G}\left\{\Phi^{-}(z)-\Phi^{+}(z)\right\}=-\frac{\left(V-V^{*}\right)}{c}$

The Plemelj formulas permit one to obtain the density function $f_{1}(t)$

$$
f_{1}(t)=\Phi^{+}(t)-\Phi^{-}(t)=-\frac{2 G\left(V-V^{*}\right)}{c\left(1-\beta_{1} \beta_{2}\right)}
$$

Note that $f_{1}(t)$ is a real constant. One possible solution is given by

$$
\Phi(z)=\frac{f_{1}}{2 i \pi} \int_{S T} \frac{d t}{t-z}
$$

With this form of solution, the displacement at infinity should be logarithmic, and not of order $O(1 / z)$ as required and thus does not respect the continuity condition on $y=0$. Thus the expression of $\Phi$ given by (39) must be modified. An adequate choice of a P-periodic, holomorphic and multivalued function is given by

$$
X(z)=\prod_{k=-\infty}^{+\infty}(z-(a+k b))^{\frac{1}{2}-\alpha}(z-(k+1) b)^{-\frac{1}{2}+\alpha}
$$

where $\alpha$ is a real number introduced in the previous section by the equation (33). Notice that this function is continuous through the stick segments, 
satisfies $X^{+}(z)=X^{-}(z)$ for every $z \in S T$ and is constant at infinity (equal to 1$)$.

It then follows

$$
\left\{\frac{\Phi^{+}(z)}{X^{+}(z)}-\frac{\Phi^{-}(z)}{X^{-}(z)}\right\}=-\frac{2 G\left(V-V^{*}\right)}{c\left(1-\beta_{1} \beta_{2}\right)} \frac{1}{X^{+}(z)}
$$

Therefore, the expression of complex potential in the stick zone is given by [26]

$$
\Phi^{s t i c k}(z)=\frac{f_{1}}{2 i \pi} X(z)\left\{\int_{S T^{+}} \frac{d t}{X^{+}(t)(t-z)}+C_{1}\right\}
$$

where $C_{1}$ is constant.

\subsection{Stick-slip solution}

The stick-slip potential is obtained by sum the two complex solutions $\Phi^{\text {stick }}$ and $\Phi^{\text {slip }}$. We obtain the following Cauchy integral with discontinuous density

$$
\Phi(z)=\frac{1}{2 i \pi} X(z)\left\{f_{1} \int_{S T^{+}} \frac{d t}{X^{+}(t)(t-z)}+f_{2} \int_{S L^{+}} \frac{d t}{X^{+}(t)(t-z)}+C_{0}\right\}
$$

where $C_{0}$ is an arbitrary constant, $f_{1}$ and $f_{2}$ are constant given by (30) and (38) respectively. Moreover, since the stress field vanishes at infinity then $C_{0}=0$.

Explicit evaluation of $\Phi(z)$ requires the knowledge of the explicit expressions of the Cauchy integrals in (42). One obtains

$$
\Phi(z)=f_{1} \frac{g}{g-1} i X(z) e^{-i \pi \alpha}\left(\frac{1}{Z(z)}-1\right)+\frac{f_{2}}{1-g}(1-X(z))
$$

where $Z(z)$ is defined by the same infinite product (34) but arranged differently, with cuts along stick zones ST (instead of cuts along slip zones for $X(z))$

$Z(z)=\cdots\left(z-b_{-(k-1)}\right)^{-\beta}\left(z-a_{-k}\right)^{\beta} \cdots \underbrace{\left(z-b_{(k-1)}\right)^{-\beta}\left(z-a_{k}\right)^{\beta}}_{Z_{k}(z)}\left(z-b_{k)}\right)^{-\beta} \cdots$

where $\beta=\frac{1}{2}-\alpha$. 
Explicit calculation gives the following analytic expression of the stick-slip potential splitted in its real and imaginary parts

$$
\begin{aligned}
\Phi(z \in S L)= & \left\{-\frac{f_{1}}{2}(1-|X(z)|)+\frac{T^{*}}{\gamma_{1}} \cos (\pi \alpha)|X(z)|\right\}+ \\
& i\left\{-\frac{f_{1}}{2} \mu \frac{\gamma_{2}}{\gamma_{1}}(|X(z)|-1)+\frac{T^{*}}{\gamma_{1}}(1-|X(z)| \sin (\pi \alpha))(45)\right.
\end{aligned}
$$

and

$$
\Phi(z \in S T)=-\frac{f_{1}}{2}-i\left\{-\frac{f_{1}}{2} \mu \frac{\gamma_{2}}{\gamma_{1}}+\frac{f_{1}}{2 \cos (\pi \alpha)}|X(z)|+\frac{T^{*}}{\gamma_{1}}(1-|X(z)|)\right\}
$$

It is worth noting that these complex functions (45) and (46) are weakly singular (i.e. square integrable) which avoids the problem of unbounded energy sources or sinks [15] at switching boundaries between stick and slip segments.

Let us remark that the nature of the mathematical singularity was explored in $[24,27]$ where it was shown that the singularity $\left(-\frac{1}{2}+\alpha\right)$ is associated to push-in test of rigid fibre in its elastic matrix material and the singularity $\left(\frac{1}{2}-\alpha\right)$ corresponds to pull-out kinematics.

\section{Results and discussion}

Let us recall that the stick-slip function $\Phi$ must behave as $O\left(1 / z^{2}\right)$ at infinity then terms of order $O(1 / z)$ must be canceled. This condition will be first imposed for the truncated product $X^{N}(z)$.

For $|z| \rightarrow \infty$, after some cumbersome calculus, one obtains

$$
\Phi_{N}^{\text {stick }}(z)+\Phi_{N}^{\text {slip }}(z)=-\frac{\beta}{z} \frac{(2 N+1)}{g-1}\left\{f_{1} g i e^{-i \pi \alpha}\left(a-b_{-1}\right)+f_{2}(b-a)\right\}
$$

Observe that this equation is independent of $N$ and thus it is sufficient to cancel terms of order $O(1 / z)$ over a single and an isolated generic stick-slip segment $\left[b_{-1}=0, a\right] \cup[a, b]$. This results in

$$
f_{1} g i e^{-i \pi \alpha}\left(a-b_{-1}\right)+f_{2}(b-a)=0
$$

or in an equivalent manner

$$
\frac{2 G\left(V-V^{*}\right)}{c\left(1-\beta_{1} \beta_{2}\right)}\left(\gamma_{1}+i \mu \gamma_{2}\right) e^{-i \pi \alpha}\left(a-b_{-1}\right)+2 T^{*}(b-a)=0
$$


Dividing (49) by $a$ and setting $b_{-1}=0$ (i.e. the stick segment starts at the origin $O$ of the coordinate frame) one obtains

$$
f_{1}\left(\gamma_{1}+i \mu \gamma_{2}\right) e^{-i \pi \alpha}-2 T^{*}(\delta-1)=0
$$

where $\delta=b / a$. Equation (50) is apparently a complex one in the form $F_{1}(\delta, c)+i F_{2}(\delta, c)=0$, where $F_{1}$ and $F_{2}$ are real, so that it should be splitted into two real equations. However, exact calculus shows that $F_{2} \equiv$ $f_{1}\left(-\gamma_{1} \sin (\alpha \pi)+\mu \gamma_{2} \cos (\alpha \pi)=0\right.$ because $\tan (\alpha \pi)=\mu \frac{\gamma_{2}}{\gamma_{1}}$. Equation (50) is thus reduced to $F_{1}(\delta, c)=0$ which provides the first relation between the wave velocity $c$ and $\delta$

$$
\frac{G\left(V-V^{*}\right)}{c\left(1-\beta_{1} \beta_{2}\right)}\left(\gamma_{1} \cos (\alpha \pi)+\mu \gamma_{2} \sin (\alpha \pi)\right)+T^{*}(\delta-1)=0
$$

The second relation between $c$ and $\delta$, which enables complete determination of these unknowns will be set further by setting the continuity condition obtained by periodicity of the total field namely, $U_{x}(b)=U_{x}(0)$, so that according to $(2), u_{x}(b)-u_{x}(0)=\frac{b V^{*}}{c}$. This permits one to have the second equation allowing complete determination of $c$ and $\delta$. This "closure" condition results in

$$
-\frac{V}{c} \delta+\left\{\frac{\left(V-V^{*}\right)}{c}-\left(1-\beta_{1} \beta_{2}\right) \cos (\pi \alpha) \frac{T^{*}}{2 G \gamma_{1}}\right\} \pi\left(\frac{1}{2}-\alpha\right) \frac{(\delta-1)}{\cos (\pi \alpha)}=0
$$

In conclusion, the unknowns of this stick-slip problem $(c, \delta)$ for given loading conditions $\left(T^{*}, V, V^{*}\right)$ are completely determined by the set of the two equations (51) and (52).

Physical quantities involved in the problem may be scaled as follows

$a=1, \quad \delta=\frac{b}{a} \rightarrow b>1, \quad \frac{T^{*}}{G} \rightarrow T, \quad \frac{V}{c_{2}} \rightarrow v, \quad \frac{V^{*}}{c_{2}} \rightarrow v^{*}, \quad \frac{c_{1}}{c_{2}} \rightarrow \bar{c}_{1}, \quad \frac{c}{c_{2}} \rightarrow \bar{c}$

We obtain the following final equations

$$
-\frac{v}{\bar{c}} b+\left\{\frac{\left(v-v^{*}\right)}{\bar{c}}-\left(1-\beta_{1} \beta_{2}\right) \cos (\pi \alpha) \frac{T}{2 \gamma_{1}}\right\} \pi\left(\frac{1}{2}-\alpha\right) \frac{(b-1)}{\cos (\pi \alpha)}=0
$$

and

$$
\frac{\left(v-v^{*}\right)}{\bar{c}\left(1-\beta_{1} \beta_{2}\right)}\left(\gamma_{1} \cos (\alpha \pi)+\mu \gamma_{2} \sin (\alpha \pi)\right)+T(b-1)=0
$$


Following results in literature [9, 10, 20], the stick-slip wave propagates from rear to front, i.e. from the right to the left in our problem. Therefore, we shall search for a wave celerity in the range $-c_{2}<c<0$ (or $-1<\bar{c}<0$ for the non dimensional equations).

The stick-slip problem $(\bar{c}, b)$, for given loading conditions $\left(T, v, v^{*}\right)$ is solved by the set of the two equations (53) and (54). This problem is highly non linear in the wave velocity $c$ and the uniqueness of the solution cannot be easily proved. Moreover its numerical solution by Newton's method for example, is inaccurate and fails because of singularities. Let us propose a simpler method to solve it. Instead of finding $(\bar{c}, b)$ for a given loading condition $\left(v, v^{*}, T\right)$ (or $\left(v, v^{*}, \tau_{x y}^{*}\right)$ since $)$, we search the solution of the following inverse problem:

Find $(v, T)$ for any given pair $(\bar{c}, b)$ and given $v^{*}$.

Therefore, one obtains a linear algebraic system

$$
\left[\pi\left(\frac{1}{2}-\alpha\right) \frac{(b-1)}{\cos (\pi \alpha)}-b\right] \frac{v}{\bar{c}}-\left[\pi\left(\frac{1}{2}-\alpha\right)(b-1) \frac{\left(1-\beta_{1} \beta_{2}\right)}{2 \gamma_{1}}\right] T=\pi\left(\frac{1}{2}-\alpha\right) \frac{(b-1)}{\cos (\pi \alpha)} \frac{v^{*}}{\bar{c}}
$$

and

$$
\left[2 \frac{\left(\gamma_{1} \cos (\alpha \pi)+\mu \gamma_{2} \sin (\alpha \pi)\right)}{\left(1-\beta_{1} \beta_{2}\right)}\right] \frac{v}{\bar{c}}+[b-1] T=2 \frac{\left(\gamma_{1} \cos (\alpha \pi)+\mu \gamma_{2} \sin (\alpha \pi)\right)}{\left(1-\beta_{1} \beta_{2}\right)} \frac{v^{*}}{\bar{c}}
$$

The system (55-56) is mathematically well-posed in the sense that we have two equations for two unknowns $(v, T)$. Note that once $T$ is known the stresses $\tau_{y y}^{*}$ and $\tau_{x y}^{*}$ are easily obtained.

The solution is straightforward and depends on the determinant of the linear system

$$
D(b, \bar{c})=\frac{(b-1)}{\gamma_{1} \bar{c}} H(b, \bar{c})
$$

where

$H(b, \bar{c})=-\gamma_{1} b+\gamma_{1} \pi\left(\frac{1}{2}-\alpha\right) \frac{(b-1)}{\cos (\pi \alpha)}+\pi\left(\frac{1}{2}-\alpha\right)\left(\gamma_{1} \cos (\alpha \pi)+\mu \gamma_{2} \sin (\alpha \pi)\right)$

It is easy to establish that $H(b, \bar{c})>0 \forall b>1$ and $\forall \bar{c} \in]-1,0[$. Thus the determinant $D(b, \bar{c})$ is strictly negative $\forall b>1$ and $\forall \bar{c} \in]-1,0[$.

We shall distinguish two cases: $v^{*}=0$ and $v^{*} \neq 0$. 


\subsection{Solution for $v^{*}=0$}

When $v^{*}=0$, equations (55-56) are reduced to a homogenous algebraic system. The later have non trivial solutions in the domain $-1<\bar{c}<0$ and $b>1$ if and only if the determinant $D(b, \bar{c})$ vanishes. However, we have checked that $D(b, \bar{c})<0$ in the domain $-1<\bar{c}<0$ and $b>1$. Therefore, only the trivial solution $v=0$ (statics) and $T=0$ (the loading at the Coulomb limit) exists.

We conclude that no solution exists in the case $v *=0$. This result agrees with the conclusion of Caroli (2000) for friction of a viscoelastic medium against a rigid half-space where no solution is found.

\subsection{Solution for $v^{*} \neq 0$}

For the case $v^{*} \neq 0$ the solution is straightforward for $v$ and $T$

$$
v=\frac{v^{*}}{1+A(b, \bar{c})}
$$

where

$$
A(b, \bar{c})=\frac{4 b \gamma_{1} \cos (\alpha \pi)}{\pi(-1+2 \alpha)\left(\mu \gamma_{2} \sin (2 \pi \alpha)+\gamma_{1}(-1+2 b+\cos (2 \pi \alpha))\right)}
$$

and

$$
\begin{aligned}
& T=-\frac{4 b \gamma_{1}\left(\gamma_{1}+\gamma_{1} \cos (2 \alpha \pi)+\mu \gamma_{2} \sin (2 \alpha \pi)\right)}{(b-1)\left(-1+\beta_{1} \beta_{2}\right)} \times \\
& \frac{v^{*}}{\bar{c}\left(4 b \gamma_{1} \cos (\alpha \pi)+\pi(-1+2 \alpha)\left(\mu \gamma_{2} \sin (\pi \alpha)+\gamma_{1}(-1+2 b+\cos (\pi \alpha))\right)\right.}
\end{aligned}
$$

Note that the solution pair $(v, T)$ exists and is unique for any wave velocity such that $-1<\bar{c}<0$, any $b>1$ and for a given $v^{*}$.

As can be noted in Eq.(22) and Eq.(23), the gradient $u_{x, x}$ and the stress $\sigma_{y y}$ are of opposite signs. Since the slip velocity has a negative singularity at $x=b$ (fig.3), the normal stress shows a positive singularity as seen in Fig.4 where $\Sigma_{y y} / G$ is plotted. Note also that, in the stick zone at the transition point with slip segments, the shear stress is singular and thus the stick 
condition (10) is violated over a very small zone, cf. fig.5. A similar result has been pointed out by Adda-Bedia and Ben Amar (2003) in analyzing the existence of a single steady-state slip pulse between two elastic media by the use of Weertman's dislocation formulation.

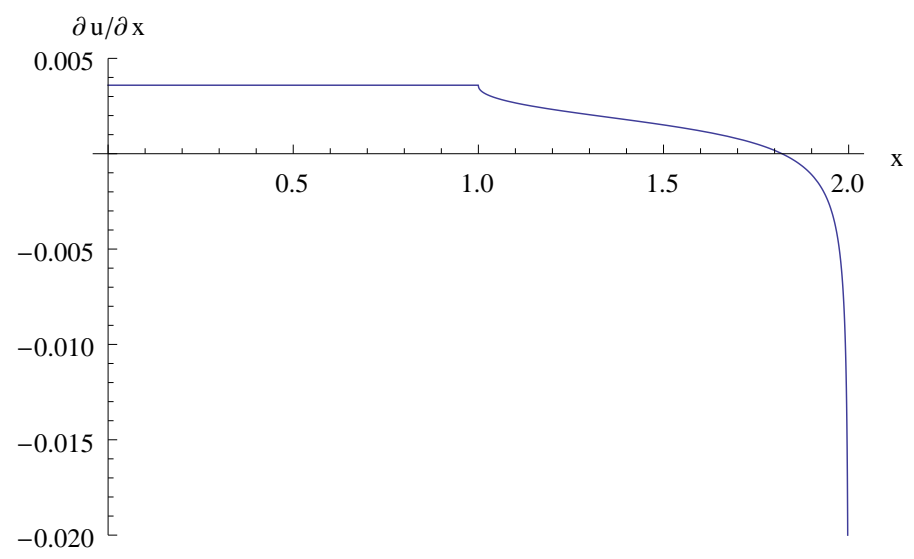

Figure 3: The gradient $u_{x, x}$ over a stick-slip segment: a negative singularity near $b=2$ with $E=2.110^{11} \mathrm{~Pa}, \rho=7850 \mathrm{Kg} / \mathrm{m}^{3}, \nu=0.3, \mu=0.5, \mu^{*}=0.4, v^{*}=0.001$ and $\bar{c}=-0.7$

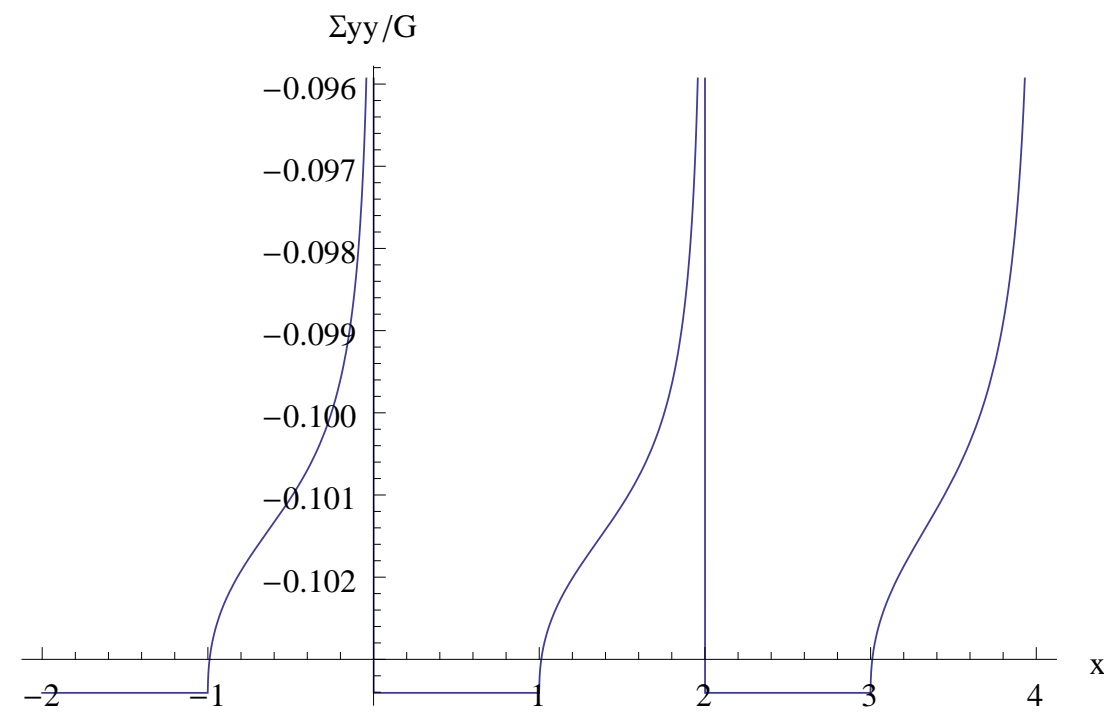

Figure 4: The normalized stress $\Sigma_{y y} / G$ (for the same numerical data as for Fig.3) over stick-slip segments exhibits a positive singularity at the transition points $S L \rightarrow S T$ 


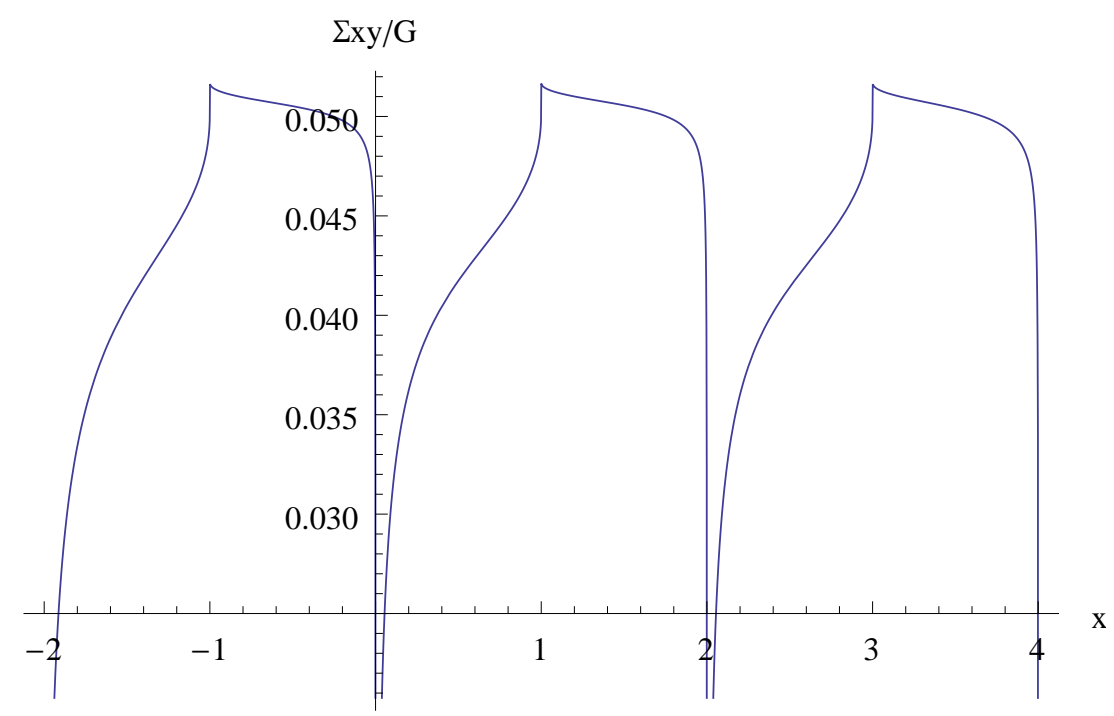

Figure 5: The normalized stress $\Sigma_{x y} / G$ (for the same numerical data as for Fig.3) over stick-slip segments shows a negative singularity at the transition points $S L \rightarrow S T$

It is worth noting that the simplicity of the inverse problem allows an easy parametric study of sensibility of the results under various conditions. We may calculate $R=$ (separation region) $/ b$ (where $\Sigma_{y y}>0$ ) and $S$ the ratio of the zone length for which (10) is violated by $b$. They are found very small for a wide range of different combinations of the problem parameters. For example, for fixed $E=2.110^{11} \mathrm{~Pa}, \rho=7850 \mathrm{Kg} / \mathrm{m}^{3}, \nu=0.3, \mu-\mu^{*}=0.2, v^{*}=0.001$ and $\bar{c}=-0.7$, it is found that $R(b=2)=610^{-5}$ and $R(b=1.05)=3.110^{-6}$ while $S(b=2)=310^{-4}$ and $S(b=1.05)=6.310^{-5}$. Besides for fixed $b=1.5, v *=0.1, c=-0.7$ and $\mu=0.7$ these ratios vary with respect to $\mu^{*}$ as follows: $R\left(\mu^{*}=0.1\right)=10^{-3}$ and $R\left(\mu^{*}=0.65\right)=3.310^{-6}$ while $S\left(\mu^{*}=0.1\right)=210^{-2}$ and $S\left(\mu^{*}=0.65\right)=6.310^{-5}$. Finally, the ratios $R$ and $S$ are practically not sensitive to variation of $v^{*}$ and $c$.

\section{Conclusion}

In this paper we have analyzed the stick-slip waves between an elastic halfspace and a rigid one under unilateral contact and dry friction. The method of representation of plane strain steady state elastodynamics by Radok's potentials has been used and the stick-slip problem is reduced to a RiemannHilbert problem. We have shown that he obtained equation can be solved in a 
closed-form under the conditions that a uniform longitudinal strain $\varepsilon_{x x}^{*}=u_{x, x}^{*}$ is considered as the consequence of the normal stress $\tau_{y y}^{*}$. If the strain $\varepsilon_{x x}^{*}$ vanishes or is ignored $\left(V^{*}=-c u_{x, x}=0\right)$ there is no solution. Furthermore, it is shown that the solution is obtained by solving an inverse procedure in which the loading conditions $(v, T)$ is determined as a function of the wave celerity $\bar{c}$, $b$ and $v^{*}$. There is no oscillatory behavior of the stress but the solution shows a crack-like behaviour: the shear stress exhibits a negative singularity while the normal stress has a positive singularity at the extremity of the slip region which also means a separation in a narrow zone. This incomplete solution is obtained for "regular-weakly singular" at the ends $a_{k}$ and $b_{k}$ respectively.

Contrary to some authors who change the friction law and adopt generally a rate-and-state friction law to overcome this difficulty, our future plans is to conserve the Coulomb friction law and to investigate at least many possible models in the future: (i) Model of stick-slip-separation (ii) Stickslip-separation-reverse slip waves as already worked out semi-analytically and numerically by Oueslati et al [28] and Nguyen et al [20] (iii) Model of stickslip with regular functions at $a_{k}$ and $b_{k}$, for which the method outlined in this paper does not work.

\section{References}

[1] Galin, L.A., 1943. Mixed problem of the theory of elasticity for the halfplane (neglecting friction). Dokl. Akad. Nauk. SSSr, 39,88-93. (In Russian)

[2] Galin, L.A., 1945. Pressure of a stamp in the presence of friction and adhesion. Prikl. Matem. I Mekh., 413-424. (In Russian)

[3] Galin, L.A., 1946. Spatial contact problems of the theory of elasticity for a stamp with circular planform. Prikl. Matem. I. Mekh., 413-424. (In Russian)

[4] Galin, L.A., 1948. Estimate of the displacement in spatial contact problems of the theory of elasticity. Prikl. Matem. I. Mekh., 413-424. (In Russian)

[5] Galin, L.A., 1948. On the pressure of a rigid body on a plate. Prikl. Matem. I. Mekh., 413-424. (In Russian) 
[6] Bui, H.D., Oueslati, A. 2010. On the stick-slip waves under unilateral contact and Coulomb friction. Annals of Solid and Structural Mechanics, $1,159-172$.

[7] Nguyen, Q.S., 2000. Stability and Nonlinear Solid Mechanics. Wiley, Chichester.

[8] Renardy, M. Ill-posedness at the boundary for elastic solids sliding under Coulomb friction. J. Elasticity, 27, 281287 (1992).

[9] Martins, J.A.C., Guimaraes, J., Faria, L.0, 1995. Dynamic surface solutions in linear elasticity and viscoelasticity with frictional boundary conditions. J. Vib. Acoust, 117, 445-451.

[10] Simoes, F.M., Martins, J.A.C., 1998. Instability and ill-posedness in some friction problems. Intr. J. Engn. Science, 36, 1265-1293.

[11] Adams, G.G., 1998. Steady sliding of two elastic half-spaces with friction reduction due to interface stick-slip. J. Appl. Mech., 65, 470-475.

[12] Ranjith, K., Rice, J.R., 2001. Slip dynamics at an interface between dissimilar materials. J. Mech. Phys. Solids, 49, 341-361.

[13] Comninou, M., Dundurs, J., 1977. Elastic interface waves involving separation. J. Appl. Mech., 44, 222-226.

[14] Comninou, M., Dundurs, J., 1978. Elastic interface waves and sliding between two solids. J. Appl. Mech., 45, 325-330.

[15] Freund, L.B., 1978. Discussion: Elastic interface waves involving separation. J. Appl. Mech., 45, 226-228.

[16] Adams, G.G., 1995. Self-excited oscillations of two elastic half-spaces sliding with a constant coefficient of friction. J. Appl. Mech., 62, 867-872.

[17] Adams, G.G., 2001. An intersonic slip pulse at a frictional interface between dissimilar materials. J. Appl. Mech. 68, 81-86.

[18] Adda-Bedia, M., Ben Amar M, 2003. Self-sustained slip pulses of finite size between dissimilar materials. J. Mech. Phys. Solids, 51, 1849-1861. 
[19] Moirot, F. Nguyen, Q.S, 2000b. An example of stick-slip wave. C. R. Mecanique, 328, 663-669.

[20] Moirot, F., Nguyen, Q.S., Oueslati, A., 2002. An example of stick-slip and stick-slip-separation waves. Eur. J. Mech. A/Solids, 22, 107-118.

[21] Nguyen, Q.S., Oueslati, A., Steindl, A., Teufel, A., Troger, H., 2008. Travelling interface waves in a brake-like system under unilateral contact and Coulomb friction. C.R. Mecanique, 336, 203-209.

[22] Caroli, C., 2000. Slip pulses at a sheared frictional viscoelasticnondeformable interface. Physical Review E, 62, 1729-1737.

[23] Radok, J.M.R., 1956. On the solution of problems of dynamic plane elasticity. Quart. Appl. Math., 14, 289.

[24] Bui, H.D., Oueslati, A., 2005. The sliding interface crack with friction between elastic and rigid bodies. J. Mech. Phys. Solids, 53, 1397-1421.

[25] Bui, H.D., Oueslati, A. 2006. Exact solutions of the interface crack between elastic and rigid bodies under contact with friction. CRAS, 332, 709-716.

[26] Muskhelishvili, N. I., 1953. Some basic problems of the mathematical theory of elasticity. Noordhoff, Groningen.

[27] Deng, X., 1994. An asymptotic analysis of stationary and moving cracks with frictional contact along bimaterial and in homogeneous solids. Int. J. Solids Struct. 31, 2407-2429.

[28] Oueslati, A., Nguyen, Q.S, Baillet, L., 2003. Stick-slip-separation waves in unilateral and frictional contact. C. R. Mecanique, 331, 133-140. 